\title{
Certified Calibration of a Cable-Driven Robot Using Interval Contractor Programming
}

\author{
Julien Alexandre dit Sandretto, Gilles Trombettoni, David Daney, and \\ Gilles Chabert
}

\begin{abstract}
In this paper, an interval based approach is proposed to rigorously identify the model parameters of a parallel cable-driven robot. The studied manipulator follows a parallel architecture having 8 cables to control the 6 DOFs of its mobile platform. This robot is complex to model, mainly due to the cable behavior. To simplify it, some hypotheses on cable properties (no mass and no elasticity) are done. An interval approach can take into account the maximal error between this model and the real one. This allows us to work with a simplified although guaranteed interval model. In addition, a specific interval operator makes it possible to manage outliers. A complete experiment validates our method for robot parameter certified identification and leads to interesting observations.
\end{abstract}

Key words: Cable-Driven Robot, Calibration, Interval Analysis

\section{Introduction}

Due to tolerances in manufacturing or assembly, the geometry of the actual manipulator does not correspond to the desired design and its theoretical kinematic model. Consequently, the performances of the manipulator, such as its accuracy, decrease. This problem could be bypassed by improving the kinematic model, i.e., by finding the actual values of its parameters. These parameters, defining the geometry of the robot (frame and platform) and actuator parts, are provided by a kinematic calibration procedure. Calibration consists in identifying model parameters through

Julien Alexandre dit Sandretto, David Daney

Coprin INRIA, France, e-mail: \{Julien.Alexandre_dit_Sandretto,David.Daney\}@inria.fr

Gilles Trombettoni

LIRMM, Univ. Montpellier 2, France, e-mail: Gilles.Trombettoni@lirmm.fr

Gilles Chabert

LINA, Ecole des Mines de Nantes, France, e-mail: Gilles.Chabert@mines-nantes.fr 
redundant information on the state of the robot provided by measurements or constraints [13]. Cable-driven robots have several interesting properties like reduced mass of moving parts (i.e., the cables), ease of reconfiguration and, more importantly, a potentially very large workspace. Nevertheless, the cost of these advantages is a complex kinematic and dynamic behavior due to the flexibility, mass and elasticity of the cables. This kind of complex numerical model, can (and often needs to) be simplified to be used in a command process or a calibration for instance. For example, a kinematic calibration with joint sensors has been reported in [3], whereas a self-calibration procedure for a planar robot is introduced in [2]. Several studies have been carried on cable-driven robot kinematics. Many of them use an approximate model for cables which considers them without mass and elasticity. The error added by this approximation has been bounded for a whole workspace and a range of usual forces in [12]. In robotics, interval analysis is used to manipulate bounded uncertainties [10, 9], or to consider the whole workspace of a robot. A recent approach called contractor programming was proposed by Chabert and Jaulin to solve different interval problems [4]. A contractor is a procedure that reduces the search space, considering generally a set of constraints to satisfy. In the face of outliers, i.e., measures that are completely wrong, a solution which satisfies all the constraints may not exist. An original interval approach for calibration was proposed by one the authors in [5]. However, outliers (along with model errors) led their method to an empty solution. This paper proposes an innovative calibration approach based on certified interval methods. The approach allows the calibration process to manage uncertainties in cable model and in measures, even in presence of outliers thanks to a recent interval operator called q-intersection. This intersection can relax some constraints to escape from outliers.

\section{Cable-driven robot}

This study is part of the CoGiRo (Conception of Giant Robot) project. The goal of this national project is to create a traveling crane with a large workspace and $n=6$ DOFs. A crane or cable-driven robot is a mechanism that controls $m=8$ cables whose length or tension provides a movement of the mobile with 6 DOFs w.r.t. a reference.

Cable-driven robot architecture: In the sketch presented in Fig. 1, the mobile (linked to the frame $\Omega_{C}$ ) is connected to the base (linked to the frame $\Omega_{O}$ ) by $m=8$ cables ( $m>n$ to be fully controllable). The $i^{\text {th }}$ cable connects the point $A_{i}$ in the base (coordinate $a_{i}$ in $\Omega_{O}$ ) to the point $B_{i}$ on the mobile (coordinate $b_{i}$ in $\Omega_{C}$ ). The pose of the mobile (defined by the position $P$ and the orientation $R$ of $\Omega_{C}$ expressed in $\Omega_{O}$ ) is directly controlled by the length and the tension of each cable. The prototype, named ReelAx8 and shown in Fig. 1, was built by the TECNALIA company (www.tecnalia.com) in collaboration with the LIRMM laboratory (www. 1 i rmm. fr). Eight cables, wound on winches, are attached by spherical joints to the eight corners of a cube shaped platform of about 40 centimeters large. 


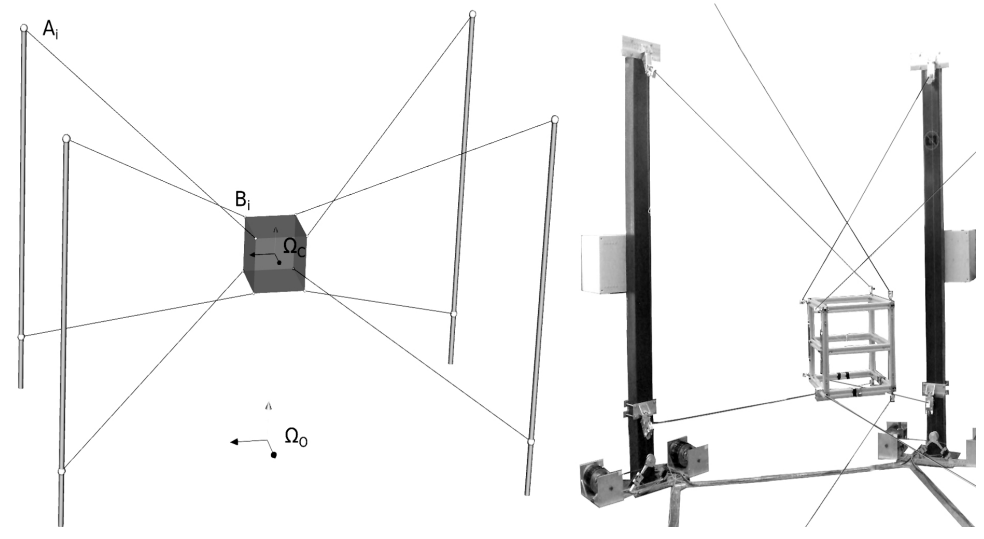

Fig. 1 A cable-driven robot sketch and a ReelAx8 picture

Four pairs of winches are fixed on posts up to three meters high arranged at the four corners of a 3 meters by 4 meters rectangle.

Interval based kinematic model: Cable-driven robots take advantage of the use of cables, allowing large workspace, light actuators compared to the possible load mass, and low cost. However, cable-driven robots suffer from the complex kinematics and dynamics of cables. A well-known realistic model often used for the kinematics of cables is proposed by Irvine in [7]. In Irvine's model, the length of a cable depends on its tension and is described by a non linear system of equations. In order to use this realistic model, we need more sensors to measure the tension in cables, but the force sensors are not accurate enough. The hypothesis considering non-elastic and mass-less cables is very useful to simplify control, modeling, calibration, etc. Moreover, it is often realistic and adds a negligible error to the robot accuracy. The majority of papers about cable-driven robots uses this hypothesis and replaces the distances between points $A_{i}$ and $B_{i}$, noted $d\left(A_{i}, B_{i}\right)$, by the length $L_{i}$, $i=1 . . m$, of unwound cables. The error between the real length of cable and the distance $d\left(A_{i}, B_{i}\right)$ has been quantified (and bounded with certification) in [12].

This method evaluates the interval cable length error $[\Delta L]=\left[\Delta L_{\min }, \Delta L_{\max }\right]$ between the realistic Irvine model $L_{\text {irvine }}$ (which depends on the tension in cables and considers mass and elasticity of cables) and the strongly simplified model $L=d(A, B)$ over the whole robot workspace and for the whole range of possible tensions in cables. An interval $[x]=[\underline{x}, \bar{x}]$ defines the set of reals $x$ s.t. $\underline{x} \leq x \leq \bar{x}$. For a given pose we can write: $\Delta L_{\min }<L-L_{\text {irvine }}<\Delta L_{\max }$

We can thus define an interval based kinematic model which deals with this modeling error:

$$
\left\|P+R b_{i}-a_{i}\right\|_{2}-\left(L_{i}+[\Delta L]\right) \supset 0, \quad i=[1 \ldots m]
$$

The simplified model approximates the realistic cable by adding the error $[\Delta L]$. Thus we can also write $L_{\text {irvine }} \in L+[\Delta L]$. This model is therefore correct because 
it contains the real one. In other terms, our interval model overestimates the real model in a guaranteed way. It is relevant for calibration because the intersection principle can reduce this overestimation.

Calibration of a Cable-driven Robot: The goal of calibration is to enhance the robot performances by the model knowledge improvement. This improvement consists in identifying the model parameters through redundant information on the state of the robot provided by measurement. In case of calibration, $N_{C}$ measurements of $P$ and $R$ are achieved, for example, with a laser tracker, and are given with tolerances. Therefore $[P]$ and $[R]$ include these tolerances, i.e., the intervals $[P]$ and $[R]$ contain all the possible values for the position and orientation. The coordinate of the point $B_{i}$ expressed in $\Omega_{O}$ at the $k^{t h}$ pose is then: $\left[b_{i}^{k}\right]=\left[P_{k}\right]+\left[R_{k}\right]\left[b_{i}\right]$. The coordinates $b_{i}$ which define the geometry of the platform are also given with manufacturing or measuring tolerances. In this paper, we focus on the $A_{i}$ coordinate identification. In practice, in the case of big cable-driven robots, the parameters $b_{i}$, which describe the platform geometry, are well known, are easy to measure and do not change, contrarily to the $a_{i}$ which change at each new reconfiguration and are not easy to be measured without a laser due to the large dimension of the robot (4 meters in our case). $\left[a_{i}\right]$ denote the coordinates of points $A_{i}$. These points are unknown, but we can bound them, even not accurately. The aim of the calibration process described in this paper is to reduce these uncertainties. The identification scheme consists of handling $m$ independent and over-constrained systems of $N_{C}$ equations:

$$
0 \in\left\|\left[b_{i}^{k}\right]-\left[a_{i}\right]\right\|_{2}-\left(\left[L_{i}^{k}\right]+[\Delta L]\right), \quad k=\left[1 \ldots N_{C}\right], i=[1 \ldots m]
$$

Due to the independence of these $m$ systems, each $a_{i}$ can be identified separately. The nature of these calibration equations, where the only variable is $a_{i}$, leads to an analogy with a thick sphere intersection problem $\left(N_{C}\right.$ thick spheres of center $B_{i}^{k}$, of diameter $\left[L_{i}^{k}\right]+[\Delta L]$ intersected in $\left.A_{i}\right)$. In the sake of clarity of pictures that follow, the problem will be illustrated by a circle (ring) intersection in a plane.

This calibration model takes into account all the bounded uncertainties on modeling, measurements and parameters. Nevertheless it could happen that a measurement is completely wrong, i.e., beyond the estimated tolerances. Several circumstances can lead to a mismeasurement, such as a sensor break down or bad environmental conditions. Such a measure is called an outlier. If it is the $c^{\text {th }}$ measure, for example, it means that:

$$
0 \notin\left\|\left[b_{i}^{c}\right]-\left[a_{i}\right]\right\|_{2}-\left(\left[L_{i}^{c}\right]+[\Delta L]\right), i=[1 \ldots m]
$$

In the presence of outliers, a classic constraint solver will fail to find a solution. In this paper, we propose to use a recent interval operator called q-intersection which can manage these outliers. 


\section{Contractor Programming and q-intersection contractor}

The calibration process suffers from two problems: the acquisition of measures and the model used for identification. The interval approach offers a rigorous representation of the uncertainties on measurements and a powerful ability to rigorously enclose the solutions. Significant theoretical and algorithmic progress have been brought to interval methods that are now mature for application [6,9]. A good introduction to the interval arithmetic can be found in these last two references. An interval solver is based on a branch \& bound. A simple solver evaluates all the functions involved in the system of constraints handled and checks if their images contain 0 . To improve this approach, contractors are used.

A contraction procedure or contractor accepts as input a box, i.e., a vector of intervals, and outputs a contracted box (while respecting certain properties detailed in [4]). A given contractor can accept any other parameter to specify it behavior, such as a system of constraints. A contractor program includes calls to several contractors applied to different boxes. The contractor program used in our calibration applies the following scheme to the system of equations modeling our measures:

$$
\text { FixP }\left(\operatorname{QInter}\left(q=75 \%, h c 4 r\left(F_{1}, \text { box }\right), \ldots, h c 4 r\left(F_{N_{C}}, \text { box }\right)\right)\right.
$$

where $h c 4 r$ [1], QInter [8] and FixP are three contractors.

- $h c 4 r$ can contract a box w.r.t. a single constraint such that no solution of the constraint is lost in the box. Using a tree representation of the constraint for accelerating the contraction, this contractor isolates every occurrence $x_{i}$ in the expression and performs a natural evaluation of the corresponding function to contract $\left[x_{i}\right]$. Applied to our ring intersection problem, $h c 4 r$ intersects the studied box with a ring corresponding to a measure.

- QInter can forget bad measures (outliers), provided that the number of good measures exceeds $q$. This contractor returns the smallest box including all the points that belong to at least $q$ of the boxes in argument. Applied to our ring intersection problem, QInter returns the box enclosing all the points in the studied box satisfying at least $q$ imprecise measures.

- FixP (FixPoint) calls the contractor in argument (here QInter) until the box reaches a quasi fixed point in terms of size. ${ }^{1}$

\section{Interval Calibration Approach}

Our tool is dedicated to calibration and uses contractor programming to find the $m$ boxes that are guaranteed to contain the points $A_{i},[i=1 . . m]$. The method is explained in details in the following.

\footnotetext{
${ }^{1}$ In our tool, the process stops if the maximal reduction on every dimension does not exceed $1 \%$.
} 


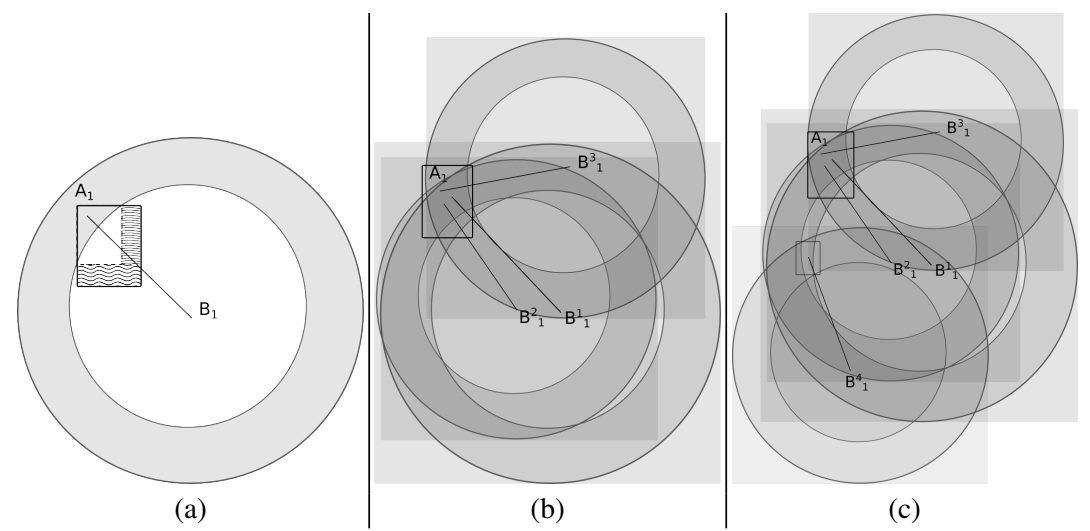

Fig. 2 (a) A pose contracting the box around $A_{1}$, (b) A circle intersection, (c) An empty circle intersection due to an outlier

Contractor for each pose: For each pose $k$ (measure of position, orientation and cable length), the $h c 4 r$ contractor reduces the size of the box including the point $A_{i}$ using the calibration constraints $F_{i, k}=\left\|\left[b_{i}^{k}\right]-\left[a_{i}\right]\right\|_{2}-\left[L_{i}^{k}\right]+[\Delta L]$. The resulting box of this contraction process is smaller than the initial box, but it is always an overestimation of the coordinate $a_{i}^{k}$ (obtained with the $k^{t h}$ pose). This is illustrated in the plane (for the sake of clarity) in Fig. 2. (a).

Calibration with q-intersection: Our calibration procedure is based on the intersection principle. The interval paradigm ensures that every pose $a_{i}^{k}$ contains the point $A_{i}$, depending on the corresponding measurement. Therefore the different boxes resulting from the different poses $a_{i}^{k}$ are intersected, thus offering a better estimation of the point $A_{i}$. In Fig.2. (b), we present the intersection of 3 rings corresponding to poses ( $B_{i}^{k}$ represents the point $B_{i}$ in the $k^{t h}$ pose). Each rectangle in grey represents a contracted box corresponding to one pose, and the final box results from the intersection of all the individual boxes.

A problem can appear in this intersection process. In practice, due to the measure acquisition process, a value may be completely wrong (e.g., $B_{1}^{4}$ in Fig. 2. (c)), even if one considered the maximal possible error allowed by the measurement device. These outliers generally lead to an empty solution for a classical intersection method, as shown in Fig. 2. (c). In order to avoid this phenomenon (trap), our calibration approach uses a q-intersection contractor. This method, presented in Section 3, can reject some poses considered as outliers (e.g., $B_{1}^{4}$ ), even if we do not know in advance which ones are outliers. This works in the calibration of our robot provided that the parameter $q$ has been set to more than $75 \%$. If the q-intersection result is nevertheless empty, additional measurements have to be achieved to obtain a correct calibration result. In a very unsettled environment, or with non accurate tools, it is also possible to choose a smaller value $q$, although the calibration process has no sense when $q$ falls below $50 \%$. 
Method experimentally applied: After a complete simulation which validated the approach, we have performed a validation with measures coming from experiment. The cable model error computed with the method presented in [12] is $[\Delta L]=[-0.6,3.7]$ millimeters. The calibration is performed with 42 measures obtained with a laser tracker. The tolerances of this tool is $\pm 1 \mathrm{~mm}$ for the position (on $x, y$ and $z$ ) and $\pm 10 \mathrm{mRad}$ for rotation (around each axis). An estimation on $a_{i}$ is given with a tolerance of $\pm 1 \mathrm{~cm}$. The calibration results are reported in Tab. 2. The

\begin{tabular}{c|c|c|c}
$b_{i}$ & $\mathrm{x}$ & $\mathrm{y}$ & $\mathrm{z}$ \\
\hline$b_{1}$ & {$[-0.112,-0.110]$} & {$[0.345,0.347]$} & {$[-0.017,-0.015]$} \\
$b_{2}$ & {$[0.241,0.243]$} & {$[0.337,0.339]$} & {$[-0.028,-0.026]$} \\
$b_{3}$ & {$[-0.171,-0.169]$} & {$[0.339,0.341]$} & {$[-0.431,-0.429]$} \\
$b_{4}$ & {$[0.298,0.300]$} & {$[0.329,0.331]$} & {$[-0.446,-0.444]$} \\
$b_{5}$ & {$[-0.179,-0.177]$} & {$[-0.031,-0.029]$} & {$[-0.429,-0.427]$} \\
$b_{6}$ & {$[0.288,0.290]$} & {$[-0.041,-0.039]$} & {$[-0.437,-0.435]$} \\
$b_{7}$ & {$[-0.118,-0.116]$} & {$[-0.027,-0.025]$} & {$[-0.004,-0.002]$} \\
$b_{8}$ & {$[0.233,0.235]$} & {$[-0.034,-0.032]$} & $[-0.015,-0.013])$
\end{tabular}

Table 1 Parameters $b_{i}$ (in meter) estimated with $\pm 1 \mathrm{~mm}$ tolerance

\begin{tabular}{c|c|c|c}
$a_{i}$ & $\mathrm{x}$ & $\mathrm{y}$ & $\mathrm{z}$ \\
\hline$a_{1}$ & {$[2.214,2.214]$} & {$[0.604,0.606]$} & {$[-2.728,-2.727]$} \\
$a_{2}$ & {$[2.199,2.219]$} & {$[0.516,0.536]$} & {$[-0.367,-0.347]$} \\
$a_{3}$ & {$[0.512,0.532]$} & {$[2.135,2.155]$} & {$[-2.711,-2.691]$} \\
$a_{4}$ & {$[0.536,0.556]$} & {$[2.193,2.205]$} & {$[-0.425,-0.405]$} \\
$a_{5}$ & {$[-1.955,-1.955]$} & {$[-0.418,-0.415]$} & {$[-2.726,-2.725]$} \\
$a_{6}$ & {$[-2.063,-2.043]$} & {$[-0.375,-0.355]$} & {$[-0.438,-0.418]$} \\
$a_{7}$ & {$[-0.310,-0.309]$} & {$[-2.020,-2.020]$} & {$[-2.727,-2.727]$} \\
$a_{8}$ & {$[-0.392,-0.375]$} & {$[-2.045,-2.043]$} & {$[-0.403,-0.398]$}
\end{tabular}

Table 2 Parameters $a_{i}$ (in meter) identified with interval method and cable model error

solution given by a least square method (described in [11]) falls outside the certified interval solution obtained with our method. For example, a classical method computes $a_{1}=(2.2046,0.5964,-2.7184)$. This means that the least square solution does not satisfy the calibration equations. Using a standard interval approach, the solution of the calibration is empty due to the presence of outliers. We can also observe that $\left[a_{3,6}\right]$ are not contracted by our method. This means that the measures are not well chosen for calibrating these parameters (identifiability problem). The measures are numerous but they do not cover all the robot workspace. Remember that in this approach, the more different correct poses we have, the smaller the final box will be. The stopping criterion is given by the time spent to make the measures, but the choice of the poses is very important [5].

\section{Discussion and conclusion}

This paper presents the first attempt to achieve a certified calibration of a cabledriven robot using an innovative contractor programming approach. Our interval 
method is robust to measurement error and outliers. Also, it permits to capture an error due to the modeling. We can use a highly simplified model for the calibration that is guaranteed to contain the realistic one (even if the latter cannot be characterized). We have employed a q-intersection contractor to obtain an outlier-resistant calibration. The obtained solution is not a point $A_{i}$, like with the least square method, but a box. This box is an overestimated enclosure of the point to be identified. We could verify that this solution is close to the one obtained by the (non certified) least square method. To summarize, this contractor programming approach holds the potential to become a key feature in certified calibration.

\section{Acknowledgment}

This work was supported in part by the French National Research Agency (ANR) under grant 2009-SEGI-018 (CoGiRo ANR project) and in part by the Région Languedoc-Roussillon under grant 115217.

\section{References}

[1] Benhamou, F., Goualard, F., Granvilliers, L., Puget, J.F.: Revising Hull and Box Consistency. In: Proc. ICLP, pp. 230-244 (1999)

[2] Borgstrom, P., Jordan, B., Borgstrom, B., Stealey, M., Sukhatme, G., Batalin, M., Kaiser, W.: Nims-pl: A cable-driven robot with self-calibration capabilities. Robotics, IEEE Transactions on 25(5), 1005 -1015 (2009)

[3] Bouchard, S.: Geometrie des robots paralleles entraines par des cables. Ph.D. thesis, Universite Laval, Quebec (2008)

[4] Chabert, G., Jaulin, L.: Contractor programming. Artificial Intelligence (2009)

[5] Daney, D., Andreff, N., Chabert, G., Papegay, Y.: Interval method for calibration of parallel robots : Vision based experiments. Mechanism and Machine Theory 41, 929-944 (2006)

[6] Hansen, E.R.: Global Optimization Using Interval Analysis. Marcel Dekker Inc. (2003)

[7] Irvine, H.M., Caughey, T.K.: The linear theory of free vibrations of a suspended cable. Proceedings of the Royal Society of London. Series A, Mathematical and Physical Sciences 341(1626), pp. 299-315 (1974)

[8] Jaulin, L.: Robust set-membership state estimation; application to underwater robotics. Automatica 45(1), 202-206 (2009)

[9] Jaulin, L., Kieffer, M., Didrit, O., Walter, E.: Applied Interval Analysis. Springer (2001)

[10] Moore, R.E.: Interval Analysis. Prentice-Hall (1966)

[11] Sandretto, J.A.D., Daney, D., Gouttefarde, M.: Calibration of a fullyconstrained parallel cable-driven robot. In: RoManSy, Paris (France) (2012)

[12] Sandretto, J.A.D., Trombettoni, G., Daney, D.: Confirmation of hypothesis on cable properties for cable-driven robots. In: Eucomes, Santander (Spain) (2012)

[13] Wampler, C., Arai, T.: Calibration of robots having kinematic closed loops using nonlinear least-squares estimation. Proc. IFToMM-jc Int. Symp. Theory Mach. Mech. pp. 153 - 158 (1992) 